CARDIOVASCULAR MEDICINE

\title{
Insulin resistance and inflammatory activation in older patients with systolic and diastolic heart failure
}

\author{
N Wisniacki, W Taylor, M Lye, J P H Wilding
}

Heart 2005;91:32-37. doi: 10.1136/hrt.2003.029652

\begin{abstract}
Objective: To evaluate insulin resistance and systemic inflammation in older patients with systolic (SHF) or diastolic heart failure (DHF).

Patients: 52 non-diabetic patients (> 70 and $<90$ years old) with chronic heart failure (CHF) and hospitalised within the previous six months for heart failure were studied, together with a control group of older healthy volunteers $(n=26)$. On the basis of Doppler echocardiographic criteria patients were classed as having SHF $(n=27)$ or DHF $(n=25)$. Main outcome measures: Fasting glucose, insulin, $C$ reactive protein, interleukin 6, and tumour necrosis factor $\alpha$ soluble receptor II (TNF- $\alpha$ SRII) concentrations were determined. Insulin resistance was estimated by the homeostasis model assessment (HOMA).

Results: HOMA index (median, interquartile range) was higher in patients with DHF $(1.77,1.06-2.26)$

than in patients with SHF $(0.97,0.81-1.85)$ or healthy volunteers $(1.04,0.76-1.44 ; p=0.01)$. After adjustment for body mass index, age, and use of angiotensin converting enzyme inhibitors, both groups of patients with $\mathrm{CHF}$ were more insulin resistant than were healthy volunteers $(p=0.02)$. C reactive protein, interleukin 6 , and TNF- $\alpha$ SRII were all significantly $(p<0.001)$ higher in patients with DHF and SHF than in healthy volunteers. All markers of systemic inflammation were independently associated with the presence of clinical CHF.

Conclusion: Insulin resistance and inflammatory activation are present in older patients with SHF and DHF.
\end{abstract}

See end of article for

authors' affiliations

Correspondence to:

Dr Nicolas Wisniacki

University Clinical

Department, The Duncan

Building, Daulby Street,

Liverpool L69 3GA, UK

n.wisniacki@liv.ac.uk

Accepted 28 March 2004

nsulin resistance (IR) has been associated with chronic heart failure (CHF) independently of its aetiology. ${ }^{1-3}$ Despite this association, left ventricular (LV) function was not correlated with insulin sensitivity in patients with systolic heart failure (SHF) or hypertension. ${ }^{24}$

Patients with CHF and preserved LV systolic function (ejection fraction $>50 \%$ ) but with diastolic dysfunction (diastolic heart failure (DHF)) constitute up to $50 \%$ of patients with heart failure, especially among older people. ${ }^{5}$ Despite its high prevalence, little is known about the pathophysiological mechanisms that contribute to diastolic dysfunction and DHF, and no data on metabolic abnormalities or evidence of systemic inflammation are available for this patient group. Diastolic dysfunction has been observed in patients without CHF but with recently diagnosed noninsulin dependent diabetes mellitus ${ }^{6}$ and in middle aged patients with impaired glucose tolerance, ${ }^{4}$ suggesting an association with insulin resistance. Furthermore, although $\mathrm{IR}^{7}$ and CHF are more prevalent in older patients, the association between IR and CHF in older patients has not previously been studied.

Both SHF and IR have been associated with evidence of systemic inflammation, as assessed by measurements of $\mathrm{C}$ reactive protein (CRP), tumour necrosis factor $\alpha$, and interleukin 6 (IL-6) concentrations. ${ }^{8}{ }^{9}$ Cytokines have been shown to have independent predictive value of mortality in patients with advanced SHF. ${ }^{8}$

An association between insulin resistance and markers of systemic inflammation has been shown in patients with and without obesity. ${ }^{10}$ However, in a small group of patients with SHF, no association between IR and tumour necrosis factor $\alpha$ was found. ${ }^{11}$ The link between markers of inflammation and IR has not been explored in either older patients or patients with DHF.

We therefore undertook a cross sectional study to test the hypothesis that older patients with CHF, whether due to systolic or to diastolic dysfunction, are insulin resistant and have evidence of systemic inflammation compared with healthy controls of similar age. We have also evaluated the relation between markers of systemic inflammation and IR in older patients with SHF and DHF.

\section{METHODS}

\section{Study patients}

Patients older than 70 years and younger than 90 years with CHF of more than six months' duration diagnosed according to the Framingham criteria ${ }^{12}$ were prospectively recruited. All patients had been hospitalised for decompensated heart failure within the previous six months, but at the time of the study were clinically stable and had been taking the same medication for at least six weeks.

Patients with diabetes or impaired fasting glucose $(>6 \mathrm{mmol} / \mathrm{l})$ were excluded. Due to potential effects on insulin sensitivity patients taking non-selective $\beta$ blockers or thiazide diuretics were excluded, as were patients with severe valvar heart disease or valve replacement. A group of control volunteers of similar age, without symptoms or signs of cardiovascular disease, and taking no medication were also studied, and were recruited from the Merseyside Registry of Healthy Volunteers held in our department. The protocol was approved by the Liverpool research ethics committee and all patients gave written informed consent.

All participants attended the Clinical Investigation Unit in the morning between 9-10 am after a 12 hour fast. A clinical evaluation was carried out including an interview, complete

Abbreviations: $\mathrm{ACE}$, angiotensin converting enzyme; $\mathrm{BMl}$, body mass index; $\mathrm{CHF}$, chronic heart failure; CRP, $\mathrm{C}$ reactive protein; $\mathrm{DHF}$, diastolic heart failure; ELISA, enzyme linked immunosorbent assay; HOMA, homeostasis model assessment; IL-6, interleukin 6; IR, insulin resistance; LV, left ventricular; NYHA, New York Heart Association; SC, standardised coefficient; SHF, systolic heart failure; TNF- $\alpha$ SRII, tumour necrosis factor $\alpha$ soluble receptor II 
Table 1 Clinical characteristics of all groups

\begin{tabular}{|c|c|c|c|c|}
\hline & Control group $(n=26)$ & DHF $(n=25)$ & SHF $(n=27)$ & p Value \\
\hline Age (years) & $76.1(3.5)$ & $80.4(4.5)$ & $79.8(5.2)$ & 0.002 \\
\hline Men & $14(53.8 \%)$ & $12(48 \%)$ & $16(59.3 \%)$ & 0.71 \\
\hline Smokers & $1(3.8 \%)$ & $1(4 \%)$ & $5(18.5 \%)$ & 0.10 \\
\hline Weight (kg) & $69.9(12.8)$ & $65.2(9.8)$ & $63.6(12.7)$ & 0.14 \\
\hline $\mathrm{BMI}$ & $24.6(3.48)$ & $25.5(3.6)$ & $23.24(2.7)$ & 0.04 \\
\hline Waist circumference $(\mathrm{cm})$ & $88.90(13.11)$ & $89.5(10.15)$ & $82.9(12.7)$ & 0.10 \\
\hline Waist to hip ratio & $0.89(0.14)$ & $0.93(0.9)$ & $0.91(0.17)$ & 0.67 \\
\hline Systolic blood pressure $(\mathrm{mm} \mathrm{Hg})$ & $137.34(17.2)$ & $139.1(23.3)$ & $126.4(22.8)$ & 0.07 \\
\hline Diastolic blood pressure $(\mathrm{mm} \mathrm{Hg})$ & $74.53(7.6)$ & $74.3(8.32)$ & 72.85 (13.4) & 0.80 \\
\hline Heart rate (beats/min) & $66.3(9.8)$ & $76.8(15.1)$ & $81.3(23.6)$ & 0.008 \\
\hline 6 minute walk test $(\mathrm{m})$ & $379.5(69.6)$ & $248.2(106.9)$ & $221.4(101.8)$ & 0.0001 \\
\hline NYHA class & & & & 0.18 \\
\hline 1 & NA & 0 & $1(3.7 \%)$ & \\
\hline$\|$ & NA & $12(48 \%)$ & $14(51.9 \%)$ & \\
\hline III & NA & $13(52 \%)$ & $9(40.9 \%)$ & \\
\hline IV & NA & 0 & $3(11.1 \%)$ & \\
\hline Cholesterol (mmol/l) & $5.38(0.97)$ & $5.32(1.24)$ & $4.69(1.08)$ & 0.03 \\
\hline $\mathrm{HDL}$ cholesterol (mmol/l) & $1.63(0.39)$ & $1.62(0.48)$ & $1.40(0.40)$ & 0.96 \\
\hline LDL cholesterol (mmol/l) & $3.27(0.70)$ & $3.10(1.00)$ & $2.73(0.99)$ & 0.07 \\
\hline Triglycerides (mmol/l) & $1.10(0.80-1.20)$ & $1.20(1.00-1.50)$ & $1.15(0.90-1.40)$ & 0.12 \\
\hline
\end{tabular}

Data are mean (SD), number (\%), or median (interquartile range).

BMI, body mass index; DHF, diastolic heart failure; HDL, high density lipoprotein; LDL, low density lipoprotein; NA, not applicable; NYHA, New York Heart Association; SHF, systolic heart failure.

physical examination, and a standard 12 lead ECG. Coronary heart disease was diagnosed if patients had a documented myocardial infarction or previous coronary revascularisation (coronary artery surgery or angioplasty). Height, weight, and waist and hip circumferences were measured. Body mass index (BMI) and waist to hip ratio were calculated.

Heart failure severity was assessed according to the New York Heart Association (NYHA) classification and all participants underwent a submaximal exercise capacity test (six minute walk test) conducted according to a standard protocol. ${ }^{13}$ Fasting venous blood samples were taken and directly put on ice. Serum and plasma samples were processed within 30 minutes and frozen at $-80^{\circ} \mathrm{C}$.

\section{Assessment of cardiac structure and function}

All participants underwent a Doppler echocardiogram evaluation with a Hewlett Packard Sonos 1000 system with a $2.5 \mathrm{MHz}$ transducer (Hewlett Packard, Andover, Massachusetts, USA) performed by the same investigator according to a standardised protocol. ${ }^{14}$

Information regarding cardiac morphology, global ventricular function, and diastolic function were recorded by $M$ mode, two dimensional, and Doppler echocardiography. LV function was evaluated by ejection fraction calculated in the four chamber view by the area-length method. M mode was used to determine LV end diastolic diameter, interventricular septum and posterior wall thickness at end diastole, and left atrial diameter at end systole. LV wall thickness (interventricular septum + posterior wall thickness), relative wall thickness ([interventricular septum + posterior wall thickness] /LV end diastolic diameter), and LV mass (0.8 [1.04 [LV end diastolic diameter $+\mathrm{LV}$ wall thickness $]^{3}-[\mathrm{LV}$ end diastolic diameter $\left.]^{3}\right]+0.6$ ) were calculated. LV mass index was calculated by dividing the value of LV mass by body surface area.

Transmitral Doppler flow was assessed by pulse Doppler recording of transmitral filling velocity in the apical four and five chamber views with the sample volume placed in the mitral valve orifice near the tips of the leaflets. Peak E velocity, peak A velocity, deceleration time, and isovolumetric relaxation time were recorded. E:A ratio was calculated with the formula peak E velocity/peak A velocity.

SHF was diagnosed when patients with clinical heart failure had an ejection fraction $<40 \%$. DHF was diagnosed when patients with clinical heart failure had an ejection fraction $>50 \%$, associated with at least two features of cardiac dysfunction according to recognised criteria ${ }^{15}$ : LV hypertrophy (interventricular septum or posterior wall thickness $>13 \mathrm{~mm}$ ), atrial enlargement (left atrial diameter $>40 \mathrm{~mm}$ ), or LV filling pattern compatible with diastolic dysfunction (E:A ratio $<0.5$ and deceleration time $>280 \mathrm{~ms}$ or isovolumic relaxation time $>105 \mathrm{~ms}$ ). Patients with an ejection fraction between $40-50 \%$ were excluded from the study.

\section{Biochemical determinations}

Glucose was analysed by the glucose oxidase method (Roche Diagnostics Ltd, East Sussex, UK) and insulin by radioimmunoassay (Mercodia AB, Sweden; coefficient of variation $2.6 \%$ ). IR was estimated by the homeostasis model assessment (HOMA) with the formula fasting insulin $(\mu \mathrm{U} / \mathrm{ml}) /$ $\left(22.5 \mathrm{X} \mathrm{e}^{-\ln (\text { fasting glucose }[\mathrm{mmol} / \mathrm{l}])}\right){ }^{16}$

CRP was measured with a high sensitivity particle enhanced immunological agglutination technique (CRP HS kit, Roche Diagnostics Ltd; detection concentration $0.03 \mathrm{mg} / \mathrm{l}$, coefficient of variation $2.7 \%$ ). Tumour necrosis factor $\alpha$ soluble receptor II (TNF- $\alpha$ SRII) and IL- 6 were measured with a sandwich enzyme linked immunosorbent assay (ELISA) (R\&D System, Oxford, UK; coefficients of variation 3.5\% and $3.6 \%$, respectively). Noradrenaline (norepinephrine) was also

Table 2 History and medication in patients with heart failure

\begin{tabular}{llll}
\hline & DHF $(\mathbf{n}=\mathbf{2 5})$ & SHF $(\mathbf{n}=\mathbf{2 7})$ & p Value \\
\hline Hypertension & $13(52 \%)$ & $15(53.8 \%)$ & 0.50 \\
Coronary heart disease & $9(36 \%)$ & $13(48.1 \%)$ & 0.37 \\
Previous MI & $6(24 \%)$ & $10(27 \%)$ & 0.37 \\
Angina & $6(24 \%)$ & $11(40.7 \%)$ & 0.24 \\
COPD & $6(24 \%)$ & $6(22 \%)$ & 0.87 \\
Atrial fibrillation & $5(20 \%)$ & $13(48 \%)$ & 0.03 \\
Spironolactone & $3(12 \%)$ & $5(18.5 \%)$ & 0.51 \\
ACE inhibitors & $9(36 \%)$ & $18(66.7 \%)$ & 0.02 \\
Digoxin & $4(16 \%)$ & $8(29.6 \%)$ & 0.32 \\
$\beta$ Blockers & $4(16 \%)$ & $2(7.4 \%)$ & 0.41 \\
Statins & $5(20 \%)$ & $8(29 \%)$ & 0.42 \\
Aspirin & $11(44 \%)$ & $18(66 \%)$ & 0.10 \\
\hline
\end{tabular}

$A C E$, angiotensin converting enzyme; COPD, chronic obstructive pulmonary disease; MI, myocardial infarction. 
Table 3 Echocardiographic evaluation

\begin{tabular}{|c|c|c|c|c|}
\hline & Control group $(n=26)$ & DHF $(n=25)$ & SHF $(n=27)$ & $p$ Value \\
\hline Ejection fraction (\%) & $62.0(14.5)$ & $59.4(8.0)$ & $30.7(6.7)$ & $<0.001$ \\
\hline $\mathrm{LV}$ end diastolic diameter $(\mathrm{cm})$ & $4.70(0.60)$ & $4.76(0.67)$ & $5.53(0.49)$ & $<0.001$ \\
\hline $\mathrm{LV}$ end systolic diameter $(\mathrm{cm})$ & $2.71(0.67)$ & $3.17(0.62)$ & $4.80(0.75)$ & $<0.001$ \\
\hline Interventricular septum $(\mathrm{cm})$ & $1.13(0.21)$ & $1.40(0.15)$ & $1.04(0.31)$ & $<0.001$ \\
\hline Posterior wall $(\mathrm{cm})$ & $1.10(0.17)$ & $1.33(0.13)$ & $1.00(0.27)$ & $<0.001$ \\
\hline Left atrial diameter $(\mathrm{cm})$ & $3.47(0.55)$ & $4.42(0.84)$ & $4.43(1.15)$ & $<0.001$ \\
\hline LV wall thickness $(\mathrm{cm})$ & $2.23(0.35)$ & $2.73(0.23)$ & $2.04(0.58)$ & $<0.001$ \\
\hline Relative wall thickness & $0.48(0.08)$ & $0.58(0.10)$ & $0.37(0.12)$ & $<0.001$ \\
\hline LV mass index $\left(\mathrm{g} / \mathrm{m}^{2}\right)$ & $111.6(28.8)$ & $159.4(32.2)$ & $143.2(27.0)$ & $<0.001$ \\
\hline \multicolumn{5}{|c|}{ Diastolic function (patients in sinus rhythm: DHF $n=20$, SHF $n=13$, and control $n=26$ ) } \\
\hline Peak E velocity $(\mathrm{m} / \mathrm{s})$ & $60.92(14.97)$ & $61.7(24.7)$ & $75.69(24.44)$ & 0.09 \\
\hline Peak $A$ velocity $(\mathrm{cm} / \mathrm{s})$ & $78.5(15.5)$ & $85.7(23.8)$ & $69.84(33.2)$ & 0.17 \\
\hline Deceleration time (ms) & $223.6(54.6)$ & $234.5(68.4)$ & $167.3(84.27)$ & 0.01 \\
\hline Isovolumic relaxation time ms) & $111.6(25.1)$ & $128.5(22.5)$ & $123.8(35.4)$ & 0.10 \\
\hline E:A ratio & $0.80(0.26)$ & $0.86(0.76)$ & $1.45(0.93)$ & 0.01 \\
\hline Deceleration time $>280 \mathrm{~ms}$ & $3(11.5 \%)$ & $6(24 \%)$ & $2(7.4 \%)$ & 0.20 \\
\hline Isovolumic relaxation time $>105 \mathrm{~ms}$ & $13(50 \%)$ & $19(76 \%)$ & $15(55.6 \%)$ & 0.13 \\
\hline $\mathrm{E}: \mathrm{A}$ ratio $<0.5$ & $2(7.7 \%)$ & $7(28 \%)$ & $2(7.4 \%)$ & 0.05 \\
\hline
\end{tabular}

measured by sandwich ELISA (IBL GmbH, Hamburg, Germany; coefficient of variation $6.5 \%$ ).

\section{Statistical analysis}

Data were checked for normality and reported as mean (SD) (95\% confidence intervals) or median and interquartile range as appropriate. Continuous variables were compared by analysis of variance and Bonferroni post hoc test for multiple comparisons. Analysis of covariance was used to compare continuous variables adjusting the result by cofactor. We used Holm's procedure to correct for multiple testing of primary outcomes measured. Student's $t$ test was performed to compare continuous variables between two groups when appropriate. Proportions between groups were compared by $\chi^{2}$ test. Correlation coefficients were calculated by Pearson's coefficient or Spearman's rank as appropriate. To obtain normally distributed variables, the HOMA index and insulin, CRP, IL-6, and TNF- $\alpha$ SRII concentrations were naturally log transformed.

Multivariate analysis of linear regression was done with the HOMA index and cytokines as the dependent variables and those variables with $\mathrm{p}<0.25$ in the univariate analysis by a stepwise selection procedure. Data were analysed with SPSS statistical software version 11.0 (SPSS Inc, Chicago, Illinois, USA).

\section{RESULTS}

\section{Demographics}

Patients with SHF and DHF were slightly older than healthy volunteers. Weight, waist circumference, and waist to hip ratio were similar in the three groups; however, BMI was lower in the SHF group than in both the DHF and control groups. Systolic and diastolic blood pressure were similar in all groups but resting heart rate was slightly higher in the SHF group (table 1).

The majority of patients with heart failure $(90 \%)$ were in NYHA class II or III and only three SHF patients were in NYHA class IV. The distance walked in the six minute walk test was similar in both groups of patients with $\mathrm{CH}$, but was significantly greater in the control group (table 1 ).

A higher proportion of patients with SHF were taking angiotensin converting enzyme (ACE) inhibitors and more were in atrial fibrillation; otherwise, the groups with CHF were similar in terms of previous cardiovascular and noncardiovascular history (table 2).

\section{Echocardiographic evaluation}

Table 3 summarises results of the echocardiographic evaluation. As expected, left atrial diameter was larger in both groups of patients with CHF than in healthy volunteers. Although LV mass was similar in both groups of patients with CHF, the end diastolic LV wall thickness and the relative wall thickness were greater in the DHF group.

Patients with SHF had a shorter deceleration time and greater E:A ratio than those with DHF and healthy volunteers. A higher proportion of patients in the DHF group had an E:A ratio $<0.5$ (table 3 ).

\section{Insulin sensitivity}

Fasting glucose concentrations were similar in all groups, whereas fasting insulin concentrations and HOMA index were significantly higher in patients with DHF than in

Table 4 Biochemical determinations

\begin{tabular}{|c|c|c|c|c|}
\hline & Control group $(n=26)$ & DHF $(n=25)$ & SHF $(n=27)$ & p Value \\
\hline Glucose (mmol/l) & $4.94(0.52)$ & $4.95(0.67)$ & $4.83(0.57)$ & 0.70 \\
\hline HOMA index (U) & $1.04(0.76-1.44)$ & $1.77(1.06-2.26)$ & $0.97(0.81-1.85)$ & $0.01^{*}$ \\
\hline Insulin (mU/L) & $4.90(3.60-6.70)$ & $8.10(4.95-10.35)$ & $4.70(3.70-7.90)$ & $0.01^{*}$ \\
\hline CRP (mg/l) & $1.50(0.10-10.30)$ & $4.5(0.40-48.20)$ & $3.30(0.30-41.10)$ & $0.002^{*}$ \\
\hline TNF- $\alpha$ SRII (ng/l) & 2161 (1964-2644) & $3273(2491-3775)$ & $3514(2884-4355)$ & $<0.001$ * \\
\hline IL-6 (ng/l) & $1.14(0.60-1.89)$ & $2.061 .14-4.10)$ & $2.78(1.84-4.89)$ & $<0.001^{*}$ \\
\hline Noradrenaline (pg/ml) & $485(204)$ & $622(220)$ & 708 (337) & 0.01 \\
\hline
\end{tabular}

Data are mean (SD) or median (interquartile range).

*Log transformed analysis of variance.

CRP, C reactive protein; HOMA, homeostasis model assessment; IL-6, interleukin 6; TNF- $\alpha$ SRII, tumour necrosis factor $\alpha$ soluble receptor II. 
Table 5 Pearson correlation coefficients showing relation between clinical and echocardiographic variables, insulin sensitivity, and markers of inflammation

\begin{tabular}{lcccc}
\hline & $\begin{array}{c}\text { HOMA } \\
\text { index }\end{array}$ & \multicolumn{1}{c}{ CRP } & \multicolumn{1}{c}{ IL-6 } & TNF- $\alpha$ SRII \\
\hline Age & -0.15 & 0.06 & 0.15 & $0.43^{* *}$ \\
BMI & $0.32^{*}$ & 0.001 & -0.03 & -0.03 \\
Waist circumference & 0.21 & 0.14 & 0.08 & 0.04 \\
Waist to hip ratio & 0.12 & $0.27^{*}$ & $0.31^{* *}$ & 0.16 \\
Ejection fraction & 0.04 & $-0.26^{*}$ & $-0.32^{* *}$ & $-0.24^{*}$ \\
E:A ratio & -0.10 & 0.23 & 0.22 & 0.018 \\
Deceleration time & 0.07 & $-0.24^{*}$ & -0.17 & -0.01 \\
LV mass index & $0.25^{*}$ & 0.06 & $0.23^{*}$ & $0.43^{* *}$ \\
Left atrial diameter & $0.23^{*}$ & -0.01 & 0.07 & 0.08 \\
6 minute walk test & -0.06 & -0.21 & $-0.35^{* *}$ & $-0.54^{* *}$ \\
\hline${ }^{*} \mathrm{p}<0.05 ;{ }^{* *} \mathrm{p}<0.01$. & & & & \\
\hline
\end{tabular}

patients with SHF and healthy volunteers (table 4). After adjustment for age, BMI, and ACE inhibitor treatment, both SHF and DHF groups had greater HOMA index than healthy volunteers, but the heart failure groups did not differ (corrected values for healthy volunteers: 0.88 , 95\% confidence interval (CI) 0.70 to 1.11 ; DHF: $1.66,95 \%$ CI 1.35 to 2.03; SHF: $1.39,95 \%$ CI 1.11 to $1.73 ; \mathrm{p}=0.001$ ). Table 5 presents univariate correlations between the HOMA index and clinical and echocardiographic variables. In a stepwise multivariate analysis of linear regression for all participants ( $\mathrm{n}=79$ ), the HOMA index was independently associated (adjusted $r^{2}=0.39, \mathrm{p}<0.001$ ) with BMI (standardised coefficient (SC) 0.27, $\mathrm{p}=0.01$ ), LV mass index ( $\mathrm{SC}=0.32$, $p=0.005)$, history of coronary heart disease ( $S C=0.22$, $\mathrm{p}=0.04)$, age $(\mathrm{SC}=-0.21, \mathrm{p}=0.04)$, and the presence of either DHF ( $\mathrm{SC}=0.46, \mathrm{p}=0.001)$ or SHF $(\mathrm{SC}=0.34$, $\mathrm{p}=0.02)$.

\section{Markers of systemic inflammation}

Serum concentrations of CRP, IL- 6 , and TNF- $\alpha$ SRII were similarly greater in patients with DHF and SHF than in healthy volunteers (table 4). After adjustment of the variables in table 4 for multiple comparisons by Holm's procedure the significantly different variables remained significant.

In univariate analysis markers of inflammation were inversely related to ejection fraction. CRP and IL-6 correlated significantly with waist to hip ratio. TNF- $\alpha$ SRII was associated with age, the six minute walk test, and LV mass index. CRP was also negatively associated with deceleration time of mitral flow (table 5).

In the stepwise multivariate analysis of all participants TNF- $\alpha$ SRII was associated independently (adjusted $r^{2}=$ $0.40, \mathrm{p}<0.001$ ) with the presence of CHF ( $\mathrm{SC}=0.27$, $\mathrm{p}=0.02)$, distance walked in the six minute walk test $(\mathrm{SC}=0.27, \mathrm{p}=0.02)$, and age $(\mathrm{SC}=0.22, \mathrm{p}=0.03)$. IL-6 was associated independently (adjusted $r^{2}=0.30$, $\mathrm{p}<0.001$ ) with the presence of heart failure ( $\mathrm{SC}=0.31$, $\mathrm{p}=0.01)$ and waist to hip ratio $(\mathrm{SC}=0.31, \mathrm{p}=0.009$ ). CRP (adjusted $r^{2}=0.24, \mathrm{p}<0.001$ ) was associated with the presence of heart failure ( $\mathrm{SC}=0.32, \mathrm{p}=0.01$ ) and waist to hip ratio $(S C=0.28, \mathrm{p}=0.02$ ).

\section{Sympathetic activation}

Noradrenaline concentrations were significantly higher in patients with SHF than in healthy volunteers. No difference was found between DHF and healthy volunteers $(p=0.19)$ (table 4). No correlation was found between the HOMA index and noradrenaline concentrations in all participants $(r=0.06, \mathrm{p}=0.58)$.
Relation between severity of heart failure, markers of inflammation, and IR

A positive correlation was found between TNF- $\alpha$ SRII $(r=0.60, \mathrm{p}<0.001)$, IL-6 $(r=0.47, \mathrm{p}<0.001), \quad$ CRP $(r=0.20, \mathrm{p}=0.07)$, noradrenaline $(r=0.25, \mathrm{p}=0.02)$, and severity of CHF according to NYHA class in patients with SHF and in patients with DHF.

In contrast, no correlation was found between the HOMA index and NYHA class $(r=0.08, \mathrm{p}=0.45)$.

\section{DISCUSSION}

We have shown for the first time that older patients with either DHF or SHF are insulin resistant compared with healthy volunteers. We have also shown a similar raised concentration of inflammatory markers in both patients with DHF and patients with SHF, which correlates well with the severity of disease.

Our results appear discordant with previous studies where patients with SHF (ejection fraction < 40\%) were more insulin resistant than patients with coronary heart disease or healthy volunteers. ${ }^{1-3}$ In our study patients with SHF were more insulin resistant than healthy volunteers after adjustment for BMI, age, and use of ACE inhibitors, suggesting that this association has been attenuated by these factors.

Lower BMI and treatment with ACE inhibitors may contribute to the greater insulin sensitivity of patients with SHF due to the well documented beneficial effect of these factors on insulin sensitivity. ${ }^{2}{ }^{17}$ Despite previous studies that reported an increase of IR with age, this is not an obligatory finding in the elderly. ${ }^{7}$ The decline in insulin action starting at age 50 years has been reported to be reversed in patients older than 80 years. ${ }^{18}$ Concordantly with these results in the very elderly we did not find a correlation between age and the HOMA index.

The relation between insulin and LV mass has been conflicting because of the heterogeneity of the populations studied. Epidemiological studies show a univariate correlation between LV mass and IR that seems to disappear after adjustment for BMI, age, or blood pressure. ${ }^{19}{ }^{20}$ Conversely, studies of patients with hypertension have shown IR to be independently associated with LV mass, ${ }^{21}{ }^{22}$ supporting our data that show an independent association between HOMA index and LV mass in patients with CHF. Hyperinsulinaemia in the face of IR stimulates myocardial cell growth, increases sympathetic activation, promotes salt and water retention, and increases vascular stiffness, all of which are associated with the development of ventricular hypertrophy. ${ }^{23} 24$

Our study showed that increased left atrial diameter is associated with greater IR, concordant with the findings of Rutter and colleagues. ${ }^{19}$ Left atrial dimension tends to be larger in patients with cardiovascular disease and this is likely to reflect left atrial pressure and volume overload in response to cardiac dysfunction associated with cardiovascular diseases. ${ }^{25}$ Left atrial pressure and increased LV end diastolic pressure are recognised early markers preceding LV hypertrophy and diastolic dysfunction in patients with hypertension. ${ }^{19} 26$

An association between Doppler parameters of diastolic dysfunction and IR has been previously shown; correlation between the peak lengthening rate of LV or E:A ratio and glucose tolerance ${ }^{47}$ or fasting insulin concentrations ${ }^{28}$ have been reported in patients with hypertension or without cardiovascular disease. We did not observe an association between fasting insulin or insulin resistance and parameters of diastolic dysfunction measured by Doppler. Parameters such as age,,$^{29}$ loading conditions, ${ }^{30}$ and heart rate ${ }^{31}$ may influence mitral inflow velocities and confer some limitation.

SHF has been proposed as an inflammatory disease; increased concentrations of cytokines and cytokine receptors 
have been shown. ${ }^{8}{ }^{32-34}$ Furthermore, cytokines are increased in patients with asymptomatic LV dysfunction, ${ }^{34}$ and increased concentrations of cytokines predict the development of $\mathrm{CHF}^{35}$ or a worse outcome in patients with clinical heart failure. ${ }^{86}$ We have observed similarly raised concentrations of inflammatory markers in patients with SHF or with DHF despite the possible effect of differences in BMI. ${ }^{37}$ We did not observe a link between inflammation, IR, and $\mathrm{CHF}$, consistent with a previous study that did not find an association between IR and tumour necrosis $\alpha$ in patients with moderate SHF. ${ }^{11}$

In our study, circulating cytokine concentrations were associated with clinical heart failure independently of the ejection fraction. However, although all cytokines correlated similarly with ejection fraction, concentrations of CRP were more variable, and IL- 6 and TNF- $\alpha$ SRII had a better correlation with indices of CHF including LV mass index and the six minute walk test. This may suggest that IL- 6 and TNF- $\alpha$ SRII are better markers than CRP of severity of CHF or diastolic dysfunction. Concordantly, TNF- $\alpha$ SRII has been reported to be the best independent predictor of mortality for CHF. $^{8}{ }^{9}$

Previous studies have provided contradictory results on the association between IR and sympathetic activation in patients with CHF. ${ }^{3}$ We found noradrenaline concentrations to be greater in patients with SHF and to be correlated with the severity of the disease according to NYHA class. However, no association was found between noradrenaline and the HOMA index. Factors other than sympathetic activation appear to be more important in determining IR in our group of patients.

In the view of the significant effect of LVH and CHD on IR and markers of inflammation we performed a post hoc analysis excluding patients known to have these conditions. In this small group $(\mathrm{n}=56)$ IR was higher in the DHF than the SHF or control groups, but this did not reach significance $(p=0.19)$. All markers of systemic inflammation were higher in patients with DHF or SHF than in healthy volunteers $(p<0.001)$. Despite the small number of patients and the nature of the analysis this may suggest that heart failure itself is a strong determinant of IR and inflammation in older patients with SHF or DHF.

The role of IR and inflammatory markers in patients with heart failure remains unclear. Their value as markers of prognosis has been established ${ }^{8}$ but therapeutic strategies targeting tumour necrosis $\alpha$ receptors have not been beneficial. $^{38}{ }^{39}$ On the other hand, the effect of insulin sensitisers in patients with mild SHF is under investigation. Our study did not try to investigate a mechanistic link between insulin resistance, inflammation, and heart failure; our objective was to describe metabolic and inflammatory abnormalities that may contribute to the understanding of DHF.

We did not measure haemodynamic factors to distinguish the diagnosis of DHF from other diseases, which is particularly difficult in the elderly. Furthermore, older healthy volunteers may have an abnormal LV filling pattern but no clinical heart failure as occurred in our group of healthy volunteers. However, all patients with CHF had been hospitalised because of heart failure within the previous six months of inclusion, and heart failure was diagnosed according to the Framingham criteria supported by positive echocardiographic criteria. Moreover, Zile and colleagues ${ }^{40}$ have shown that DHF can be diagnosed without haemodynamic measurements or parameters that reflect diastolic dysfunction.

In summary, this study has shown that patients with SHF or DHF share not only clinical features of the syndrome but also metabolic abnormalities and inflammatory activation that have previously been observed only in SHF. Whether this reflects common aetiological factors or is a consequence of CHF remains uncertain but such findings are of clinical relevance, as they may predict prognosis. Interventional studies targeting insulin resistance and inflammatory activation in CHF independent of LV function, including patients with DHF, may help determine whether modulation of these factors can improve outcomes in these patients.

\section{ACKNOWLEDGEMENTS}

The authors gratefully acknowledge the help and support from Mrs Pam Aimson and Mrs Chris Manning (heart failure specialist nurses), Miss Jayne Oakes, Mrs J Devine, and Mrs J Usher (for their help with the biochemical analyses), and the valuable statistical advice of Mrs Susanna Dodd.

This study was supported by a grant from the Institute of Human Ageing, The University of Liverpool. Dr Wisniacki is the recipient of a fellowship from Rehabilitation and Acute Medicine for the Elderly (RAMSE), Royal Liverpool and Broadgreen University Hospitals.

\section{Authors' affiliations}

N Wisniacki, J P H Wilding, Diabetes and Endocrinology Research Group, Department of Medicine, University of Liverpool, Liverpool, UK W Taylor, Department of Clinical Chemistry, University of Liverpool M Lye, Geriatric Medicine, University of Liverpool

\section{REFERENCES}

1 Swan JW, Walton C, Godsland IF, et al. Insulin resistance in chronic heart failure. Eur Heart J 1994;15:1528-32.

2 Swan JW, Anker SD, Walton C, et al. Insulin resistance in chronic heart failure: relation to severity and etiology of heart failure. J Am Coll Cardiol 1997;30:527-32.

3 Paolisso G, De Riu S, Marrazzo G, et al. Insulin resistance and hyperinsulinemia in patients with chronic congestive heart failure. Metabolism $1991 ; 40: 972-7$.

4 Nagano N, Nagano M, Yo Y, et al. Role of glucose intolerance in cardiac diastolic function in essential hypertension. Hypertension 1994;23:1002-5.

5 Senni M, Tribouilloy CM, Rodeheffer RJ, et al. Congestive heart failure in the community: a study of all incident cases in Olmsted County, Minnesota, in 1991. Circulation 1998;98:2282-9.

6 Di Bonito P, Cuomo S, Moio N, et al. Diastolic dysfunction in patients with non-insulin-dependent diabetes mellitus of short duration. Diabet Med 1996; 13:321-4.

7 Paolisso G, Tagliamonte MR, Rizzo MR, et al. Advancing age and insulin resistance: new facts about an ancient history. Eur J Clin Invest 1999:29:758-69.

8 Deswal A, Petersen NJ, Feldman AM, et al. Cytokines and cytokine receptors in advanced heart failure: an analysis of the cytokine database from the vesnarinone trial (VEST). Circulation 2001;103:2055-9.

9 Ferrari R, Bachetti T, Confortini R, et al. Tumor necrosis factor soluble receptors in patients with various degrees of congestive heart failure. Circulation 1995;92:1479-86.

10 Hotamisligil GS, Shargill NS, Spiegelman BM. Adipose expression of tumor necrosis factor-alpha: direct role in obesity-linked insulin resistance. Science 1993;259:87-91.

11 Doehner W, Rauchhaus M, Godsland IF, et al. Insulin resistance in moderate chronic heart failure is related to hyperleptinaemia, but not to norepinephrine or TNF-alpha. Int J Cardiol 2002;83:73-81.

12 McKee PA, Castelli WP, McNamara PM, et al. The natural history of congestive heart failure in the Framingham study. N Engl J Med 1971;285: 1441-6.

13 Guyatt GH, Sullivan MJ, Thompson PJ, et al. The 6-minute walk: a new measure of exercise capacity in patients with chronic heart failure. Can Med Assoc J 1985;132:919-23.

14 Schiller NB, Shah PM, Crawford M, et al. Recommendations for quantitation of the left ventricle by two-dimensional echocardiography. American Society of Echocardiography committee on standards, subcommittee on quantitation of two-dimensional echocardiograms. J Am Soc Echocardiogr 1989;2:358-67.

15 Cleland JGF, Tendera M, Adamus J, et al. Perindopril for elderly people with chronic heart failure: the PEP-CHF study. Eur J Heart Fail 1999;1:211-6.

16 Matthews DR, Hosker JP, Rudenski AS, et al. Homeostasis model assessment: insulin resistance and beta-cell function from fasting plasma glucose and insulin concentrations in man. Diabetologia 1985;28:412-9.

17 Vuorinen-Markkola H, Yki-Jarvinen H. Antihypertensive therapy with enalapril improves glucose storage and insulin sensitivity in hypertensive patients with non-insulin-dependent diabetes mellitus. Metabolism 1995:44:85-9.

18 Barbieri M, Rizzo MR, Manzella D, et al. Age-related insulin resistance: is it an obligatory finding? The lesson from healthy centenarians. Diabetes Metab Res Rev $2001 ; 17: 19-26$ 
19 Rutter MK, Parise H, Benjamin EJ, et al. Impact of glucose intolerance and insulin resistance on cardiac structure and function: sex-related differences in the Framingham heart study. Circulation 2003; 107:448-54.

20 Rheeder P, Stolk RP, Mosterd A, et al. Insulin resistance syndrome and left ventricular mass in an elderly population (the Rotterdam study). Am J Cardiol 1999:84:23-6, A9.

21 Lind L, Andersson PE, Andren B, et al. Left ventricular hypertrophy in hypertension is associated with the insulin resistance metabolic syndrome. $J$ Hypertens 1995; 13:433-8.

22 Verdecchia P, Reboldi G, Schillaci $G$, et al. Circulating insulin and insulin growth factor-1 are independent determinants of left ventricular mass and geometry in essential hypertension. Circulation 1999;100(:1802-7.

23 Westerbacka J, Wilkinson I, Cockcroft J, et al. Diminished wave reflection in the aorta: a novel physiological action of insulin on large blood vessels. Hypertension 1999;33:1118-22.

24 Young ME, McNulty P, Taegtmeyer $\mathrm{H}$. Adaptation and maladaptation of the heart in diabetes. Part II. Potential mechanisms. Circulation 2002;105:1861-170

25 Benjamin EJ, D'Agostino RB, Belanger AJ, et al. Left atrial size and the risk of stroke and death. The Framingham heart study. Circulation 1995;92:835-41.

26 Lemme C, Lindvall K, de Faire U. Changes in cardiac diastolic dimensions precede hypertrophy in early stages of hypertension. J Hum Hypertens 1998; 12:679-83.

27 Ferrara A, Barrett-Connor EL, Edelstein SL. Hyperinsulinemia does not increase the risk of fatal cardiovascular disease in elderly men or women without diabetes: the Rancho Bernardo study, 1984-1991. Am J Epidemiol 1994; 140:857-69.

28 Okura $\mathrm{H}$, Inoue $\mathrm{H}$, Tomon $\mathrm{M}$, et al. Impaired glucose tolerance as a determinant of early deterioration of left ventricular diastolic function in middle-aged healthy subjects. Am J Cardiol 2000;85:790-2, A9

29 Gardin JM, Arnold AM, Bild DE, et al. Left ventricular diastolic filling in the elderly: the cardiovascular health study. Am J Cardiol 1998;82:345-51.

30 Choong CY, Herrmann HC, Weyman AE, et al. Preload dependence of Doppler-derived indexes of left ventricular diastolic function in humans. J Am Coll Cardiol 1987;10:800-8
31 Harrison MR, Clifton GD, Pennell AT, et al. Effect of heart rate on left ventricular diastolic transmitral flow velocity patterns assessed by Doppler echocardiography in normal subjects. Am J Cardiol 1991;67:622-7.

32 Levine B, Kalman J, Mayer L, et al. Elevated circulating levels of tumor necrosis factor in severe chronic heart failure. N Engl J Med 1990;323:236-41

33 Munger MA, Johnson B, Amber IJ, et al. Circulating concentrations of proinflammatory cytokines in mild or moderate heart failure secondary to ischemic or idiopathic dilated cardiomyopathy. Am J Cardiol 1996;77:723-7.

34 Aukrust $P$, Ueland $T$, Lien $E$, et al. Cytokine network in congestive heart failure secondary to ischemic or idiopathic dilated cardiomyopathy. Am J Cardiol 1999:83:376-82.

35 Vasan RS, Sullivan LM, Roubenoff R, et al. Inflammatory markers and risk of heart failure in elderly subjects without prior myocardial infarction: the Framingham heart study. Circulation 2003;107:1486-91.

36 Torre-Amione G, Kapadia S, Benedict C, et al. Proinflammatory cytokine levels in patients with depressed left ventricular ejection fraction: a report from the studies of left ventricular dysfunction (SOLVD). J Am Coll Cardiol 1996:27:1201-6.

37 Anker SD, Ponikowski PP, Clark AL, et al. Cytokines and neurohormones relating to body composition alterations in the wasting syndrome of chronic heart failure. Eur Heart J 1999;20:683-93.

38 Chung ES, Packer $\mathrm{M}$, Lo $\mathrm{KH}$, et al. Randomized, double-blind, placebocontrolled, pilot trial of infliximab, a chimeric monoclonal antibody to tumor necrosis factor-alpha, in patients with moderate-to-severe heart failure: results of the anti-TNF therapy against congestive heart failure (ATTACH) trial. Circulation 2003;107:3133-40.

39 Krum H. Tumor necrosis factor-alpha blockade as a therapeutic strategy in heart failure (RENEWAL and ATTACH): unsuccessful, to be specific. J Card Fail 2002;8:365-8.

40 Zile MR, Gaasch WH, Carroll JD, et al. Heart failure with a normal ejection fraction: is measurement of diastolic function necessary to make the diagnosis of diastolic heart failure? Circulation 2001;104:779-82.

\section{IMAGES IN CARDIOLOGY}

\section{End stage ischaemic heart failure}

A 53 year old man presented with exertional dyspnoea during daily activities. He was a current smoker and had a positive exercise test. Angiography showed a significant left main (LM) stenosis (panel A, white arrow) and a 70\% narrowing of the circumflex artery (CX). The left anterior descending (LAD) and right coronary artery were occluded. Left ventricular (LV) function was poor and showed a large calcified anterior wall aneurysm (panel A and panel C, black arrow, end systolic frame). In a hybrid strategy, unprotected LM angioplasty with stenting was successfully performed (panel B, arrow). After three and 12 months, no angiographic restenosis was found Echocardiographic follow up confirmed improved wall motion. Thereafter, surgical resection of the anterior aneurysm was performed and arterial bypass grafts were anastomosed to the LAD and marginal branch of the CX. At 24 months follow up, LV function was excellent (panel D) and the patient had resumed his work at a local service delivery.

G A J Jessurun J G Grandjean F Zijistra g.jessurun@thorax.azg.nl
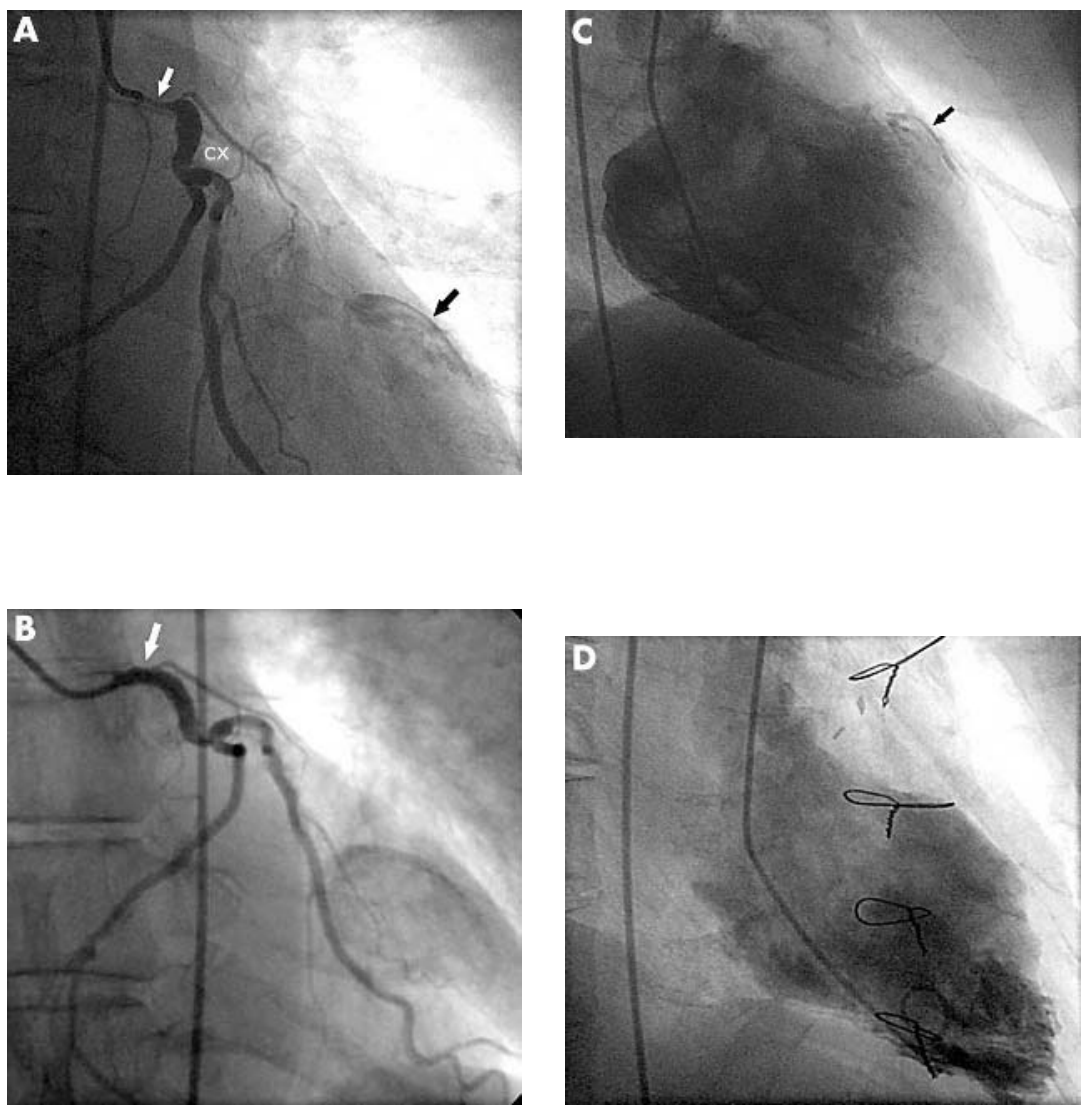